\title{
January 2018 Critical Care Case of the Month
}

Theodore Loftsgard, APRN, ACNP

\author{
Department of Anesthesiology and Critical Care \\ Mayo Clinic Minnesota \\ Rochester, MN USA
}

\section{History of Present IIIness}

The patient is a 51-year-old woman admitted with a long history of progressive shortness of breath. She has a long history of "heart problems". She uses supplemental oxygen at 1 LPM by nasal cannula.

\section{Past Medical History, Social History and Family History}

She also has several comorbidities including renal failure with two renal transplants and a history of relatively recent RSV and CMV pneumonia. She is a life-long nonsmoker. Her family history is noncontributory.

\section{Physical Examination}

- Vital signs: Blood pressure $145 / 80 \mathrm{~mm} \mathrm{Hg}$, heart rate 59 beats/min, respiratory rate $18, \mathrm{~T} 37.0^{\circ} \mathrm{C}, \mathrm{SpO} 291 \%$ of $1 \mathrm{LPM}$.

- Lungs: Clear.

- Heart: Regular rhythm with G 3/6 systolic ejection murmur at the base.

- Abdomen: unremarkable.

- Extremities: no edema

Which of the following should be performed?

1. Brain naturetic peptide (BNP)

2. Chest x-ray

3. Echocardiogram

4. Electrocardiogram

5. All of the above 


\section{Correct! \\ 5. All of the above}

The etiology of her shortness of breath is not well-defined based on the information provided. Her BNP was elevated at $200 \mathrm{pg} / \mathrm{ml}$ (normal $<74 \mathrm{pg} / \mathrm{ml}$ for a 51-year-old woman). Her electrocardiogram shows dagger-like septal $Q$ waves in the lateral and inferior leads and left ventricular hypertrophy. Chest x-ray shows cardiomegaly and enlarge pulmonary arteries bilaterally. Echocardiogram shows severe left ventricular outflow tract obstruction with a resting gradient of $159 \mathrm{~mm} \mathrm{Hg}$ with moderate to severe mitral valve regurgitation due to systolic anterior motion of the mitral valve. She has pulmonary hypertension and marked left atrial enlargement.

Which of the following are indicated?

1. Cardiology consultation

2. Digitalis to improve cardiac output

3. Diuretics for severe congestive heart failure

4. 1 and 3

5. All of the above 


\section{Correct! \\ 1. Cardiology consultation}

Review of her outside medical records, which were not available at her initial presentation, revealed a history of long-standing obstructive hypertrophic cardiomyopathy $(\mathrm{HCM})$. She has also had a fairly recent cardiac catherization which shows an absence of significant coronary artery disease, marked septal hypertrophy with a gradient across the gradient of $<150 \mathrm{~mm}$ and moderately severe mitral valve regurgitation. Mitral valve regurgitation often accompanies severe $\mathrm{HCM}$ due to anterior movement of the mitral valve.

The goal of therapy for HCM is to reduce the gradient across the hypertrophied septum (1). This can be done with beta-blockers and calcium channel blockers. Anticoagulants are often added to prevent thromboembolic phenomena. She was on each of these. In HCM the use of inotropic drugs such as digoxin should be avoided since they can make the outflow gradient worse. Similarly, nitrates and sympathomimetic amines should be avoided except in those patients with concomitant coronary artery disease. Diuretics should be used with caution because of their potential adverse effect on the LV outflow gradient and ventricular volume. The BNP is only modestly elevated and consistent with the modest elevation usually seen with HCM.

Which of the following should be considered?

1. Cardiovascular surgery consultation

2. Oral prostanoid therapy for pulmonary artery hypertension

3. Permanent pacemaker implantation

4. 1 and 3

5. All of the above 


\section{Correct! \\ 1. Cardiovascular surgery consultation}

Patients with HCM often have arrthymias. These include tachyarrthymias which can be associated with sudden death and bradyarrthymias which might require pacemaker implantation. She had not history of arrthymias, and therefore, a pacemaker is not indicated. Disopyramide (Norpace) is often added to decrease ventricular and supraventricular arrhythmias by raising the threshold for atrial and ventricular ectopy (1). In addition, disopyramide decreases the inotropic state of the left ventricle, decreases diastolic dysfunction and increases LV compliance, and thereby reducing the pressure gradient across the LV outflow tract. Our patient was receiving disopyramide. Amiodarone is often used in $\mathrm{HCM}$ and is the only agent proven to reduce the incidence and risk of cardiac sudden death (1). It is very effective at converting atrial fibrillation and flutter to sinus rhythm.

Although patients with severe HCM often have pulmonary artery hypertension, the hypertension is due to the mitral valve disease accompanies HCM.

Pulmonary vasodilators have little or no role at present.

Cardiovascular surgery is consulted and recommends a septal myectomy since the patient is on maximal medical therapy and has severe and progressive symptoms. She underwent septal myectomy and had an estimated blood loss of $1270 \mathrm{ml}$ and intraoperatively received crystalloid: $4300 \mathrm{~mL}$; packed red blood cells: 2 units; fresh frozen plasma (FFP): 4 units; cell saver: $634 \mathrm{~mL}$; platelets: 1 unit; and ultrafiltrate: $650 \mathrm{~mL}$.

She was transferred to the ICU for vasopressor weaning and diuresis and anticoagulated post-operative. She was diuressed and was only 1 liter positive and her weight had increased only $1.2 \mathrm{kgs}$ by her third post-operative day. However, on post-op day 4 she developed increasing shortness of breath with mild chest and an increase in her pulse from the 60's to the 90's. Her serum creatinine had also gradually risen to $2.7 \mathrm{mg} / \mathrm{dL}$.

Which of the following are likely causes of her shortness of breath?

1. Cardiac tamponade

2. Myocardial infarction

3. Pneumothorax

4. 1 and 3

5. All of the above 


\section{Correct!}

\section{1 and 3}

Myocardial infarction is always possible and she has undoubtedly had some myocardial injury with her myectomy. However, myocardial infarction from coronary artery disease is fairly unlikely given her lack of significant coronary artery disease preoperatively and post-operative anticoagulation.

Which of the following should be performed at this time?

1. Beside ultrasound

2. Cardiac catherization

3. Chest X-ray

4. 1 and 3

5. All of the above 


\section{Correct!}

\section{1 and 3}

The most likely causes of her shortness of breath after a myectomy is fluid overload but this seems inconsistent with her fluid status. Pneumothorax is a consideration as is cardiac tamponade. Cardiac catherization is not indicated. Chest radiography (Figure 1) and bedside ultrasound were performed (Figure 2).

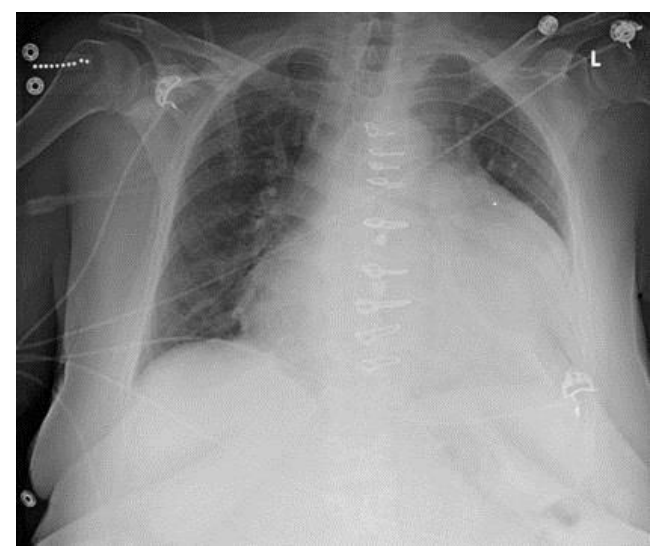

Figure 1. Portable chest radiograph.

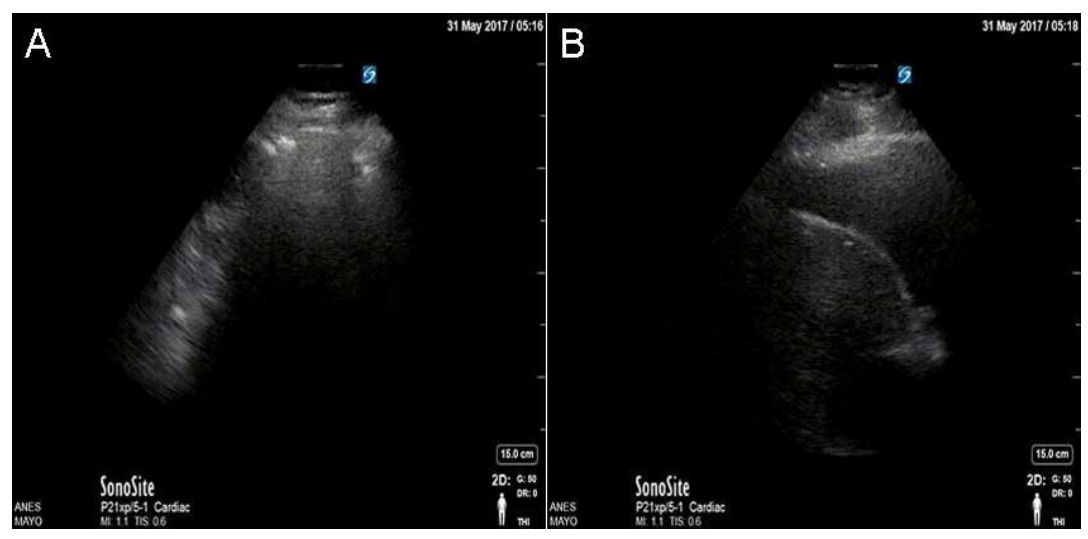

Figure 2. A: Static image from bedside ultrasound. Posterior chest, left side. Video showed the presence of lung sliding. B: Static image from bedside ultrasound. Left apical view.

Her inferior vena cava was slightly enlarged at $1.9 \mathrm{~cm}$ (normal $1.2-1.7 \mathrm{~cm}$ ).

What is the cause of the patient's shortness of breath?

1. Left atrial thrombus

2. Pericardial effusion

3. Pneumothorax

4. 1 and 3

5. All of the above 


\section{Correct! \\ 2. Pericardial effusion}

The presence of lung sliding is normal (2). The absence of lung sliding indicates a pneumothorax and is a sensitive sign on beside ultrasound. The inferior vena cava is not small indicating the patient is not volume depleted. There is a large pericardial effusion (Figure 3).

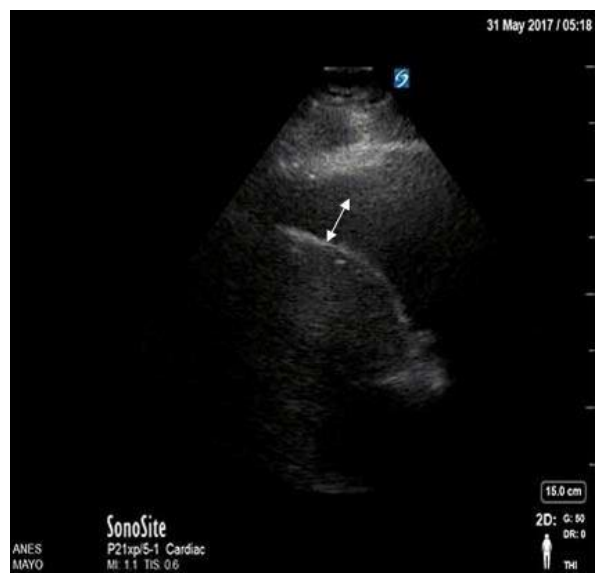

Figure 3. Bedside ultrasound. Left apical view showing a large pericardial effusion (arrow).

Other bedside ultrasound views showed collapse of the right atrium and ventricle consistent with cardiac tamponade. Pericardiocentesis was performed with the removal of $850 \mathrm{ml}$ of serosanguineous fluid. There was an almost immediate resolution of the patient's dyspnea and her vital signs returned to normal. Her creatinine decreased over 3 days and she was discharged to home.

\section{References}

1. Shah SN. Hypertrophic cardiomyopathy. Medscape. January 5, 2016. Available at: https://emedicine.medscape.com/article/152913-overview (accessed 12/22/17).

2. Lee SM, Chu GT. Ultrasound for critical care physicians: lung sliding and the seashore sign. Southwest J Pulm Crit Care. 2014;9(6):337-40. [CrossRef] 